\title{
A Discussion with Freeman Dyson
}

Michael Th. Rassias (University of Zürich, Switzerland)

Note: Just a few days before this article was printed, we were all saddened by Freeman Dyson's passing on February 28, 2020. The article below is published in its original form.

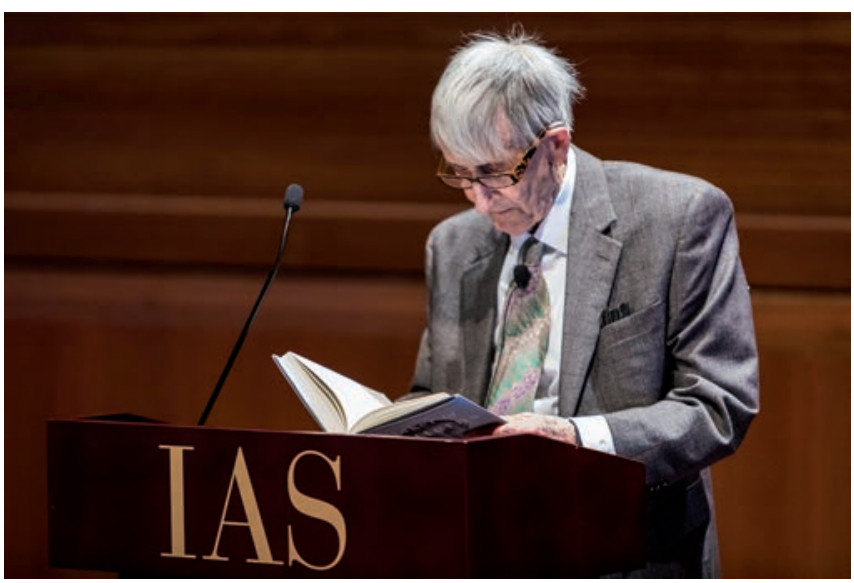

Freeman Dyson presenting his new book Maker of Patterns. (Photo: Dan Komoda/Institute for Advanced Study, Princeton, NJ, USA)

Freeman J. Dyson, born in 1923 in Crowthorne in Berkshire, England, is a world-famous American theoretical physicist and mathematician, whose academic stature is that of a historical figure of science. At the beginning of his career he worked as a civilian scientist for the Royal Air Force in World War II. In 1945 he obtained his B.A. degree in mathematics from Cambridge University. $\mathrm{He}$ had a job as an instructor at Imperial College from 1945-1946, and in 1947 he went to Cornell University as a graduate student, where he worked with Hans Bethe and Richard Feynman. Subsequently, he was a member of the Institute for Advanced Study, Princeton, from 1948-1949 and a research fellow at the University of Birmingham from 1949-1951. He then became professor at Cornell University, where he remained until 1953. Surprisingly, he was made professor at Cornell notwithstanding the fact that he did not have and never actually obtained a Ph.D. (a topic also discussed with him below). In 1953 he became a permanent professor at the Institute for Advanced Study, Princeton, where he has remained throughout the rest of his career.

Freeman Dyson has made numerous profound contributions in a broad spectrum of subjects of mathematics and physics, among which is the unification of the three versions of quantum electrodynamics invented by Richard Feynman, Julian Schwinger and Shin'ichirō Tomonaga. His work and lectures on Feynman's theories played an integral role in making them understandable to physicists of the time, and this helped Feynman's work to be accepted by the academic community. His work on this subject impressed J. Robert Oppenheimer and had an impact on him being offered a permanent position at the Institute for Advanced Study, Princeton.

Among his participation in many diverse and important projects, it is also worth mentioning that in 1958 he was a member of the design team under Edward Teller for a small and really safe nuclear reactor used throughout the world in hospitals and universities for the production of medical isotopes.

Dyson's passion has always been to explore problems through which mathematics can be usefully applied. His span of scientific interests and his everlasting appetite for exploration have lead him to investigate problems not only in mathematics, physics and their interconnections, but also in other subjects such as biology.

During his career, he has been bestowed with a plethora of awards and distinctions, including becoming a Fellow of the Royal Society (1952), receiving the Lorentz Medal (1966), the Wolf Prize (1981), the Enrico Fermi Award (1993), the Templeton Prize (2000), the Henri Poincaré Prize (2012), to name just a few.

Honoured and humbled to be surrounded by such pillars of science during my time as a visiting researcher at the Program in Interdisciplinary Studies of the Institute for Advanced Study, Princeton, I had the great privilege of first meeting Freeman Dyson in around 2015. Since then, I have had the opportunity to spend some time with him, hoping to absorb some of his wisdom. Inspired by his accomplishments, and always spellbound by his beautiful tales of the numerous interesting events of his life, the idea arose for the following discussion to be shared with the readers of the EMS Newsletter.

You started your academic career as a pure mathematician, worked with Davenport for a while and among other things proved Minkowski's conjecture in four dimensions using methods from various areas of mathematics. You later turned to physics. At which age did you decide to become a mathematician?

I met Davenport during the year 1945-1946 when I had a job at Imperial College as an instructor. He was at University College, so I was not officially his student. He generously supplied me with problems to work on, hard enough to be challenging but not impossibly hard. First was the Siegel conjecture that every algebraic number can be approximable by rational $(p / q)$ with accuracy not better than $q$ to the power two-plus-epsilon. I failed to prove it and the conjecture was later proved by Klaus Roth. Second was the Minkowski conjecture in four dimensions, which I succeeded in proving, and used as a thesis for a research fellowship at Trinity College Cambridge. I remained a life-long friend of Davenport.

I think my choice of pure mathematics for a career was mostly the result of reading Men of Mathematics by 


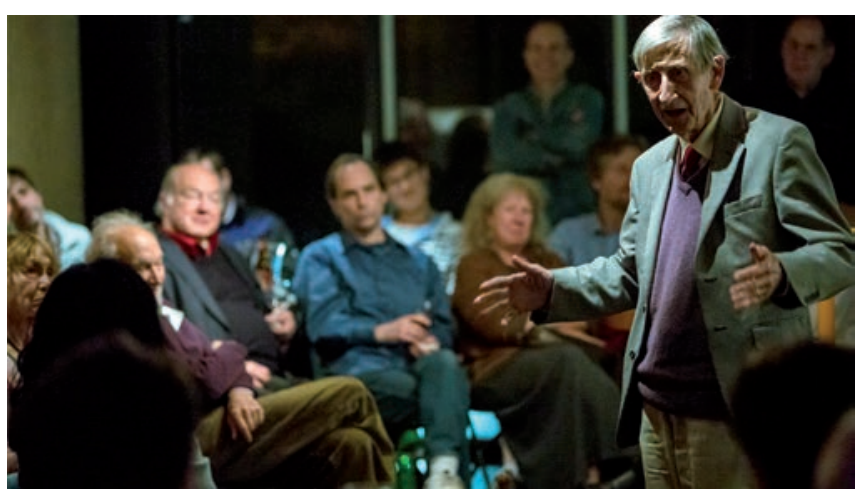

Freeman Dyson giving a lecture at the Institute for Advanced Study. (Photo: Dan Komoda/Institute for Advanced Study, Princeton, NJ, USA)

Eric Bell at the age of 13. I was doubly lucky, as Bell's book was newly published in 1937, and it was given to me as a high-school prize by Winchester College. The book is a collection of mathematical biographies, bringing mathematicians to life as real people, and explaining their work with enough technical detail to bring their ideas to life.

\section{What made you later move towards physics?}

The move to physics happened when I moved to Trinity College in 1946. There I met Nicholas Kemmer, a worldclass theoretical physicist who was also a dedicated teacher. He taught me most of what I needed to know to become a physicist. I saw that my nineteenth-century mathematics would be useful for practical problems in physics, while I remained ignorant of the more abstract ideas that were then dominating pure mathematics. It would be much more exciting to join the crowd exploring the mysteries of nuclear and particle physics than to remain in the small world of number theory. As a physicist I had no difficulty in finding funds to visit the United States where the particle physics revolution was already in full swing.

Was there a specific paper, book, lecture, or even a theorem you came across that had a lasting impact on you to the extent that it made you chose to become a scientist, rather than - say - a musician like your father? The book that most influenced my choice of career was Men of Mathematics. My father was a composer and conductor and administrator. He became director of the Royal College of Music in 1938. He had the wisdom to see that I had no musical talent and never tried to push me into music. He always encouraged me to follow my own path in science.

You never followed a doctoral program in order to obtain a Ph.D. In this subject you also have some interesting views which are fairly divergent from the norm. I was lucky to be educated in England at a time when the Ph.D. was not required as an entrance ticket to an academic career. I was always opposed to the Ph.D. system as it became more and more rigid in later years. It was a system designed for a small population of German students in the nineteenth century and was well suited to their needs. It is totally unsuited to the needs of a large and diverse population of students in the twenty-first century. It is especially harmful to women, who have to deal with the biological clock of child bearing while the rigid and lengthy $\mathrm{Ph}$.D. system is eating up their best years. It is one of the main reasons why talented women drop out of academic careers. It is also harmful to young people of all genders who do not happen to belong to wealthy or professional-class families. It has the effect of increasing and perpetuating the inequality between rich and poor in modern societies.

Is there a mathematician who influenced you the most? Either through your mutual collaboration or interaction or even by studying his/her work? I remember you mentioning A. Besicovitch in the past as one such case, who happened to also be a close friend of yours.

The mathematician who influenced me most was Besicovitch. I was lucky to arrive at Trinity College as an undergraduate at age 17 in 1941, when there were hardly any advanced students. The old and famous mathematicians, Hardy, Littlewood, Besicovitch, lavished their time and attention on the few advanced students who were there. I quickly became a personal friend of the famous mathematicians and especially with Besicovitch, who was the owner of the billiard table. I played billiards mostly with Besicovitch, but also with Hardy. Besicovitch gave me hard mathematical problems to work on and took me with him for long walks around Cambridge, on which only Russian was spoken.

Being an analyst and analytic number theorist, I was fascinated when you first told me in the past that you actually knew G.H. Hardy rather well and had even spent time with him playing billiards at Cambridge. Would you like to share some memories from your time with him, as he is known for his particular personality. Hardy gave wonderful lectures on function theory and analysis to a group of four students sitting around a small table. He prepared his lectures with great care, so that the discussion came to a dramatic climax just as the hour ended. He included stories about the personal lives and idiosyncrasies of the famous mathematicians that he had known. He talked fast and with a wealth of detail, so that I had to listen to every word. He spoke for three hours a week without ever repeating a lecture. He was fiercely opposed to the Tripos examination system that dominated Cambridge in those days just as the Ph.D. system does today. He tried and failed to abolish the Tripos just as I tried and failed to abolish the Ph.D.

\section{You also think that Hardy is partly to be blamed for} Ramanujan's death, if I remember well?

The story of Ramanujan's illness only became clear after his death. What is clear is that he died of amoebic hepatitis contracted in India before he came to England. Amoebic hepatitis was a well-known disease, which any doctor specialised in tropical medicine would have recognised. It was already then a curable disease with an 


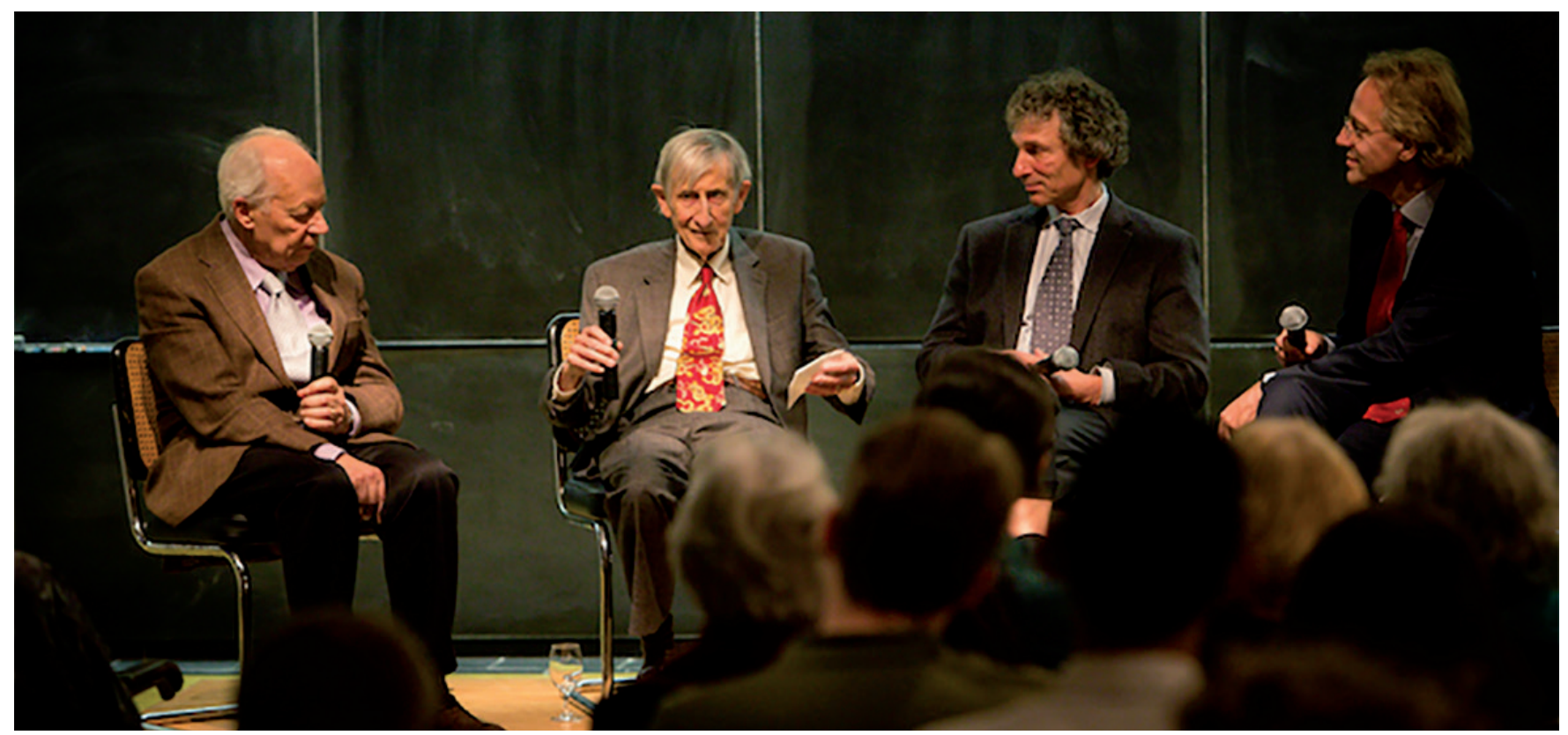

From left to right: Enrico Bombieri, Freeman Dyson, Dan Rockmore, Robbert Dijkgraaf. (Photo: Andrea Kane/Institute for Advanced Study, Princeton, NJ, USA)

effective chemical antidote. Hardy was responsible for Ramanujan's well-being when he became sick in England. Hardy never had him examined by a specialist in tropical medicine. The local doctors in Cambridge misdiagnosed his disease as tuberculosis and treated it with standard tuberculosis treatment, which was obviously ineffective. Hardy saw Ramanujan chronically sick for several years but never seemed to take the problem seriously. He enjoyed Ramanujan as a brilliant mind and an active collaborator, but did not care for him as a human being. That is a harsh verdict, but I think it is justified by the evidence.

On a humorous note, if - like in the case of Ramanujan - there were a supreme being that could hand you solutions to important problems in your sleep, and say that you could have only one such dream, the solution to which problem would you like to see?

I find it unattractive to have a dream giving me the solution of a deep mathematical or physical problem such as the Riemann hypothesis or the value of the fine structure constant. That would be too cheap. Nature gave us these problems as tests of our imagination, and to have them cheaply solved would be a loss of the mystery and beauty of Nature. So I would choose a historical question that deals with events that are lost in the past. Where and when did the first life appear on our planet? Where and when did the first human language emerge?

For a brief period of your life you lived in Zürich, where you also got to know W. Pauli. Would you like to say a few words about your interaction with him?

I was lucky to live in Zürich in the summer of 1951 when Pauli and I were the only two regular visitors at the ETH institute of theoretical physics. Pauli liked to go for walks after lunch and often invited me to join him. He loved to talk about all kinds of problems from physics to psychoanalysis, and I loved to listen. Among many other topics, we discussed the question whether the perturbation series expressing the interactions between particles in quantum electrodynamics are convergent or divergent. If the series converges, it proves the theory to exist as a well-defined mathematical object. If the series diverges, it proves that the theory is ill-defined and mathematically non-existent. I then believed that the series converged and Pauli believed that it diverged. As a result of our conversations, I found a simple proof that Pauli was right and I was wrong. I was profoundly grateful to Pauli for forcing me to confront the unwelcome truth.

Since Pauli was a quantum physicist, this reminded me of another topic. What are your views on the possibility of creation of quantum computers, as well as on the advent of areas such as quantum cryptography in our efforts to be protected against the possible use of quantum computers towards breaking current cryptosystems?

Quantum computers is not the right name for them. The right name is quantum subroutines. They are physical objects with quantum behaviour that processes information faster than classical objects can do. They must be connected to classical computer systems that provide input and output and interaction with human operators. The quantum subroutines are theoretically powerful and will probably be practically useful. They still need a massive engineering development before we can set limits to their capabilities. One of the important applications will be to cryptography. A quantum crypto-system will be theoretically more secure than a classical crypto-system. But that does not mean that the quantum crypto-system is more secure in the real world. In the real world, crypto-systems are usually broken by exploiting human 


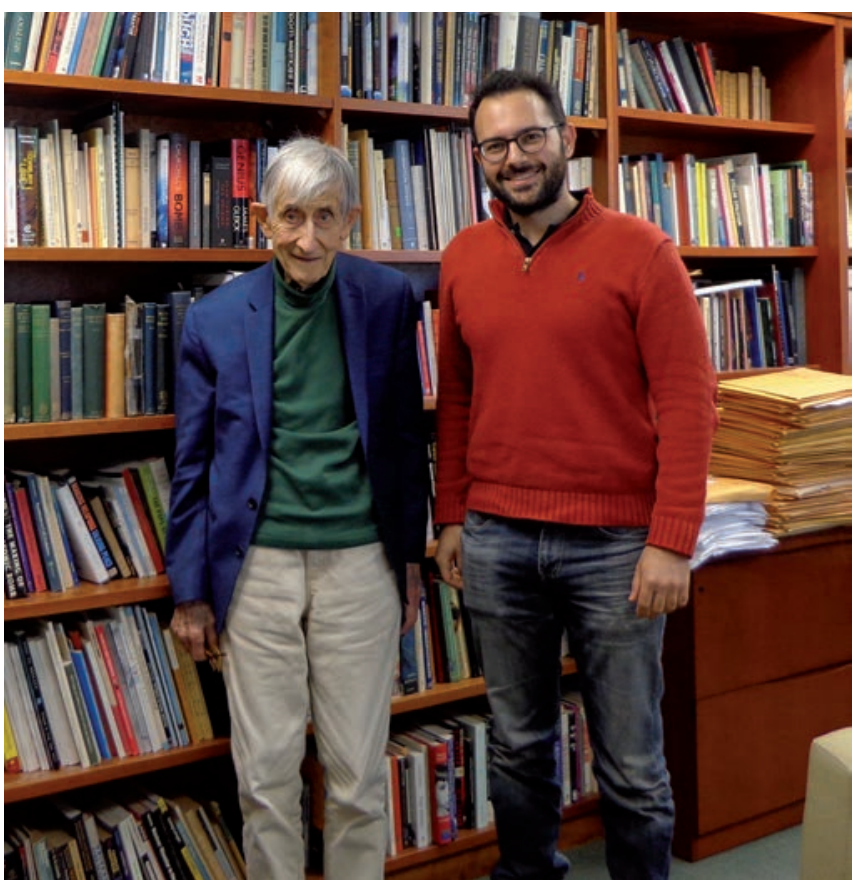

Freeman Dyson (left) at his office at the Institute for Advanced Study, Princeton, with Michael Th. Rassias, ca. March 2017.

errors in the day-to-day operations of the users. Human errors will probably be as prevalent in quantum systems as they are in classical systems. So long as humans are involved in the practical use of crypto, no system will be secure against hackers.

An amusing thing you mentioned to me in a past discussion of ours related to the important physicist $E$. Teller, whom you also knew, was that to be with Teller one had to know how to act with children. Why is that? Edward Teller worked with me on the design of a nuclear reactor called TRIGA, which was much safer than other reactors. Teller was a brilliant scientist with an abundant supply of ideas. He was also a prima donna who threw temper tantrums like a five-year-old if anyone dared to oppose him. So our collaboration worked very well. Every day Edward would suggest a new crazy idea, I would explain why his idea would not work, and he would throw a tantrum. Then the next day that idea was forgotten and a new crazy idea was suggested. In this way we converged on a design that actually worked. The collaboration worked well because I had a lot of experience dealing with five-year-old kids at home. The reactor was a commercial success and we sold 75 of them, some of them still working sixty years later.

From all the people you have met in your life, who has astonished you the most?

As it happens, the two people who impressed me the most were both scientists, Richard Feynman and Stephen Hawking. Both were physicists. This is probably not an accident. They lived at a time when physics was attracting a large fraction of the most brilliant young people, with new experiments and theories and revolutionary discoveries rushing ahead. Now, seventy years later, physics has slowed down, while astronomy and biology have speeded up. Now I talk with astronomers and biologists more than I talk with physicists.

What is the first thing that comes to mind when you are thinking of the word "physics"?

When I think of physics today, I think of the monstrous instruments like the Large Hadron Collider which cost billions of dollars to build and can only do one experiment in ten years. I also think of fashionable theories that are never tested because they do not make verifiable predictions. If I were young today, I would not choose physics as my line of work.

One may state that mathematics has witnessed great expansion during the last, say, one hundred years, with many different areas emerging and various methods discovered, bridging seemingly different fields. How do you see the future of mathematics in that respect? Do you think that interdisciplinarity might be the theme of the future, for example?

The most beautiful feature of mathematics is its unpredictability. Progress comes in big jumps, sometimes unifying the whole subject with new ideas, sometimes diversifying the whole subject with new problems. The only thing I know for sure about the future is that the next big jump will be a surprise. I do not try to guess how it will go.

Many scientists from a broad spectrum of areas have expressed various views, opinions and even fears about the possible future consequences of the advancement of Artificial Intelligence (AI), in connection to the so-called “AI-control problem". As someone who has witnessed paradigm shifts in science which defined new eras, what are your views about the possible important advances in AI which might affect human lives altogether?

Artificial Intelligence is a huge subject and I cannot summarise the prospects for its future. The clearest view of it was published in the book The Human Use of Human Beings' by Norbert Wiener in 1950. Wiener showed amazing insight in his vision of the good and bad effects of AI. He predicted the failure of our society to find benefits to humanity from the good effects and remedies for the evil. Seventy years later, his verdict, that humans competing with machines will become slaves, is being proved correct. His remedies are nowhere being applied on a scale adequate to the size of the problems. I cannot do better in defining the problems than Wiener did in 1950. 